\title{
EVIDENCE OF MOTILE TRAPS IN BYBLIS
}

\author{
DR GREGORY ALLAN •Birmingham•United Kingdom・greg_w_allan@yahoo.com
}

\author{
Introduction
}

Byblis Salisb. is a small genus of carnivorous plants with adhesive traps in the Lamiales family Byblidaceae Domin. There are two perennial species (B. gigantea Lindl. and B. lamellata Conran \& Lowrie) with restricted ranges in Western Australia, where they experience a Mediterranean climate. The critically endangered $B$ gigantea is endemic to the Swan River drainage area, now entirely within the Perth metropolitan area, whilst $B$. lamellata is restricted to the coastal region North of Perth. The genus also contains six currently recognized annual species (B. aquatica Lowrie \& Conran, B. filifolia Planch., B. guehoi Lowrie \& Conran, B. liniflora Salisb., B. pilbarana Lowrie \& Conran, and $B$. rorida Lowrie \& Conran) which inhabit the tropical and semi-arid regions of Northern Australia. The genus also extends to the island of New Guinea (Lowrie 2013; McPherson 2010). All species are found in substrates which are very nutrient-poor (Lowrie 2013; McPherson 2010) and share habitats with representatives of other genera of carnivorous plants (particularly Drosera, but also Utricularia and Nepenthes). Although there are important morphological differences between the Byblis species, all share the same basic structure in that they produce stems from which radiate filiform leaves. Another feature common to all species is the ability to produce fast concentrated growth in response to seasonal rainfall. (Bourke, pers. comm.). It has been observed on many occasions that all Byblis species play host to Miridae bugs from the genus Setocoris (Bourke, pers. comm.). A mutualistic relationship has been proven to exist between another viscid plant genus Roridula and a different genus of the family Miridae, Pameridea (Anderson \& Midgley 2003). A similar relationship is strongly suspected in Byblis (Lowrie 2013; Cross et al. 2018).

The basic structures which facilitate carnivory in Byblis are well-established. Virtually all aboveground parts of the plant, including the stems, pedicels, and sepals, are covered liberally in glands of two types. The first type is "stalked glands", each of which is furnished with a droplet of secreted mucilage and is positioned atop a stalk (often referred to as a "tentacle"), which consists of a single elongated cell (Darwin 1875; Lloyd 1942) and can be up to $2.6 \mathrm{~mm}$ in length (McPherson 2010), although length varies across species and plant organ.

The glands of the second type are far smaller and are positioned in rows within grooves in the epidermis (Lloyd 1942). They are believed to be responsible for secretion of digestive fluids and absorption of digested matter (Cross et al. 2018). Although these glands are frequently referred to as "sessile glands", this classification is erroneous, as each rests upon an even smaller stalk cell, so does not emerge directly from the epidermis (Lloyd 1942; McPherson 2010). Accordingly, they can more appropriately be called "digestive glands".

These details notwithstanding, the carnivorous syndrome in Byblis has been under-studied relative to that of other carnivorous plants. Although it has long been recognized that the glands and cellular structure of Byblis appear to be clearly adapted to facilitate secretion and absorption of the kind associated with the capture of prey and sequestration of nutrients (Darwin 1875; Lloyd 1942), the carnivorous nature of the genus has been disputed. Bruce (1905) observed apparent digestion of albumen by B. gigantea, but Lloyd (1942) was unable to perceive evidence of digestion of carmine fibrin during a two-week period within which he had access to live specimens of the 
same species. Moreover, Hartmeyer (1997) concluded that B. liniflora does not produce proteases on the basis that the plants (unlike specimens of various species (and one hybrid) of the genus Drosera) did not digest the gelatine layer of strips of photography film attached to the lamina, even when provided with stimulation 12 hours previously in the form of a $10 \%$ yeast solution. Accordingly, at least some species have on occasion been indicated as not "truly" carnivorous by some authors (see generally D'Amato 1998;

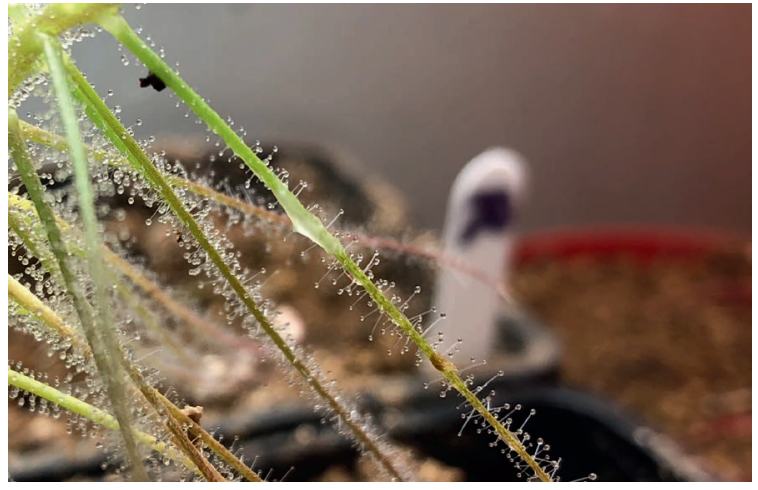

Figure 1: Cultivated Byblis liniflora with cheese that is in the process of being digested. Rice 2006).

In 2005, however, Hartmeyer (2005) used the aforementioned film-strip tests to provide evidence of protease production by B. filifolia, and Płachno et al. (2006) used enzyme labelled fluorescence to demonstrate phosphatase secretion by the digestive glands of $B$. liniflora. Additionally, the author of this article has obtained tentative evidence of protease production by $B$. gigantea and B. lamellata by using Hartmeyer's film-strip tests, and casual observations on the basis of the experiments detailed below showed apparent evidence of digestion of animal proteins in all currently recognized species (see cheese on B. liniflora in Fig. 1). Most compellingly, however, Skates (2016) deployed stable isotope techniques on B. gigantea, B. liniflora, B. filifolia, and B. rorida to demonstrate conclusively that these species are able to sequester significant quantities of nitrogen from captured prey. Although more research is required regarding the precise composition of the digestive fluids of Byblis, and of the factors which provoke their secretion, it may be postulated that the failures to detect digestion may be attributable to the fact the secretions of the digestive glands, but not the stalked glands, contain digestive enzymes. Moreover, it may well be that certain types of chemical stimulation are necessary to provoke secretion of digestive enzymes.

The traps of Byblis have been classified as non-motile ("passive") since the earliest investigation of their carnivorous properties, which was performed by Darwin using dried specimens of $B$. gigantea (Darwin 1875). Darwin posited that the unicellular nature of the stalks (called "pedicels" by Darwin) of $B$. gigantea rendered gland motility impossible because "no instance is known of unicellular structures having any power of movement" (Darwin 1875, p 344). He took the view that prey captured by the stalked glands would "...probably sink down besmeared with the secretion and rest on the small sessile glands" (Darwin 1875, p 344). Darwin's classification of Byblis as passive carnivores has essentially been accepted ever since, both in scientific works (Lloyd 1942; Juniper et al. 1989; Lowrie 1998, 2013; Ellison \& Adamec 2018) and popular texts (Slack 1979, 1986; D’Amato 1998; Rice 2006; McPherson 2008, 2010). It should be noted that Darwin undertook no experiments to investigate the process of carnivory in Byblis, and that he only studied dried plant material of a single perennial species, presumably for want of access to living plants.

There is some minor discord regarding the precise process of carnivory in Byblis. According to McPherson (2010, p 966), upon stimulation from captured prey, both the stalked and digestive glands produce "copious secretions [which cause] it to become affixed directly to the leaf blade and in contact with the digestive, enzyme-secreting sessile glands". In this respect, the carnivory of Byb- 
lis has often been thought similar to that of the monotypic genus Drosophyllum, a carnivorous plant which has some superficial morphological similarities to Byblis and which has been demonstrated to capture prey using secretions from immobile stalked glands, and to effect digestion and absorption via sessile glands (Cross et al. 2018). Notably, the carnivorous syndrome in Drosophyllum has been investigated far more thoroughly than that of Byblis. For the purposes of this article, it is particularly pertinent to note that Darwin (1875) conducted experiments on live plants which proved that its stalked glands are incapable of movement. A slight variant of the process in Byblis has been suggested whereby secretions from the stalked glands are primarily responsible for producing a "pool of fluid" in which "the prey is dissolved" (Lowrie 1998, p 16), and that the sessile glands are responsible solely for absorption of the products of digestion (Lowrie 1998; Bourke \& Nunn 2012).

\section{A rationale for the research in this paper}

There are certain respects in which the orthodox account of passive carnivory in Byblis does not stand up to scrutiny. Byblis possess morphological characteristics which suggest that, despite Darwin's insinuation to the contrary, gravity is unlikely in many instances to play a key role in the migration of captured prey from the stalked glands to the digestive glands. In Byblis, the stalked glands are most densely concentrated on the underside of the leaves, and observations of cultivated plants show that this is a frequent site of prey-capture (see also Slack 1979, p 96). Observations of specimens of $B$. filifolia, $B$. liniflora, and $B$. rorida at natural location indicate that the most common prey items are small flying insects which may be trapped and digested on any part of the leaf, including the underside (Bourke, pers. comm) (see Fig. 2). Moreover, at least in B. filifolia and B. rorida, captured and digested prey are often found adhered to the vertical stems, perhaps a consequence of doomed attempts to escape. (Bourke, pers. comm.) (see Fig. 3 left). It is also relevant to note that newly unfurled leaves are often more or less upright, whilst older leaves, especially in the annual species, usually radiate horizontally or semi-erectly from the stem. Consequently, prey is often captured in such a manner that any migration of that prey onto the leaf surface must be in an upward or horizontal direction (Fig. 3 right). Only towards the end of their active life do leaves radiate predominantly downwards so as to maximize exposure of the upper leaf surfaces and encourage prey to be captured in a position which allows for it to migrate towards the digestive glands in a downward direction, as described by Darwin.

The notion that copious secretions may be predominantly responsible for bringing prey into contact with the leaf surface and digestive glands thereon is also inconsistent with the morphology of most Byblis species. This can be seen when comparing Byblis with the superficially similar Drosophyllum. The latter genus has the glands and leaves arranged in a similar manner to those of Byblis, and the two genera have been described as exhibiting essentially the same

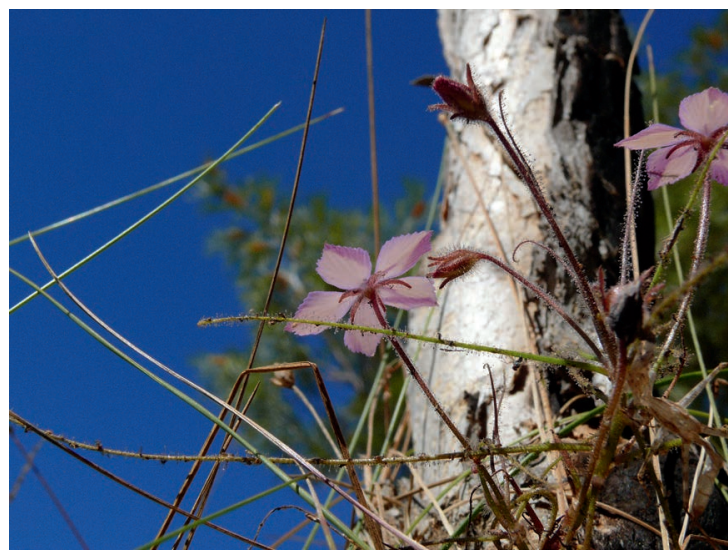

Figure 2: Prey adhered mainly to the underside of leaves of wild Byblis filifolia (photo by Andreas Fleischmann). 

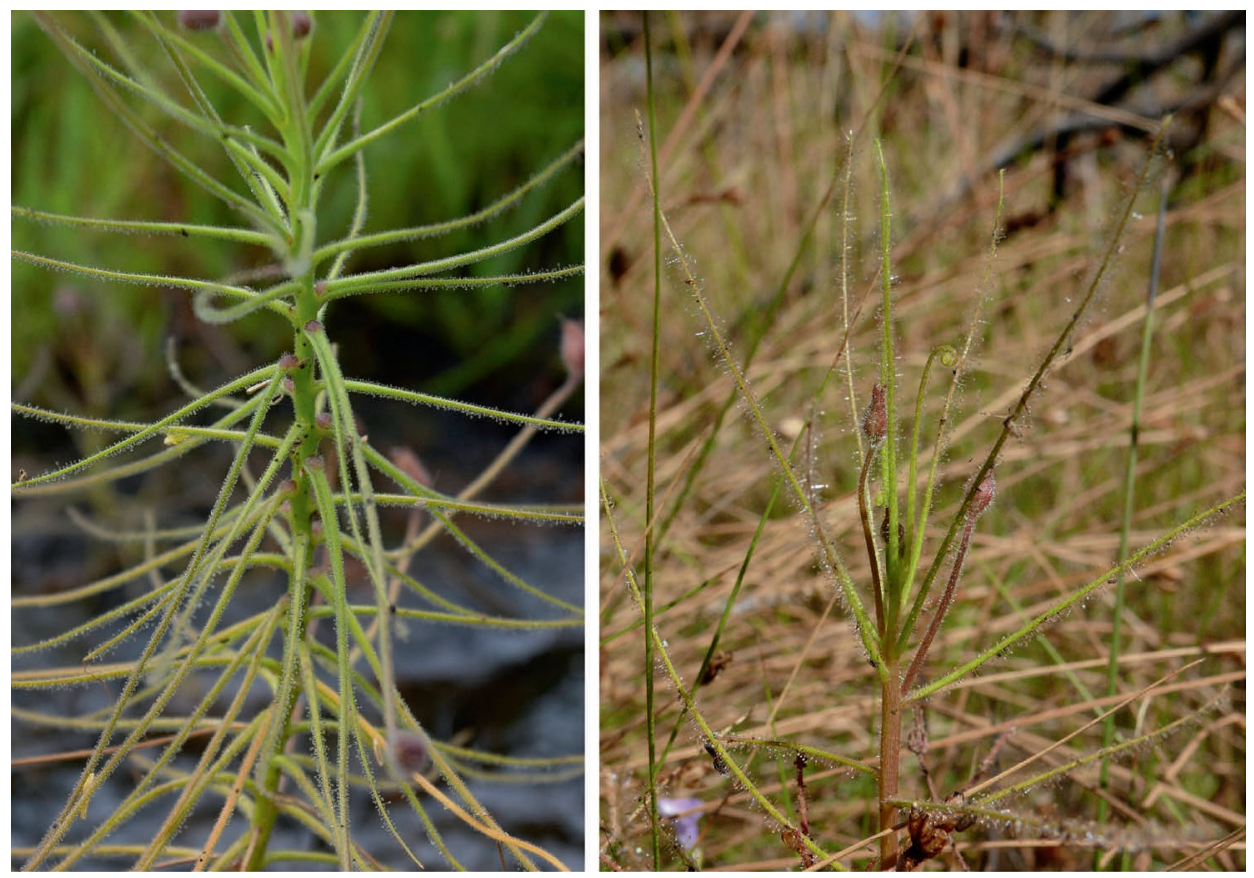

Figure 3: Wild Byblis rorida with prey adhered to stem (left, photo by Greg Bourke); wild Byblis filifolia with many lower leaf surfaces well-positioned to capture prey (right, photo by Andreas Fleischmann).

process of trapping and digestion (Darwin 1875; McPherson 2008, 2010; Slack 1979). In fact, the structure of the traps of the two genera is extremely different, and Byblis is not morphologically suited to utilizing the mode of carnivory that has been proven in Drosophyllum. Most crucially, Drosophyllum stalks, unlike those of Byblis, are multicellular and vascularized, and produce mucilage continuously (Lloyd 1942). The stalked glands of Drosophyllum are very short, so that captured prey is always within very close proximity to the leaf surface and the digestive glands. Thus, copious mucilage secretions from stalked glands provide a ready medium through which digestive fluids can travel the short distance from the leaf surface to the prey. This arrangement also produces ideal conditions for prey to be drawn into contact with the digestive glands by capillary action.

In contrast, Byblis stalks are not vascularized, and are thus unsuitable for continuous mucilage secretion. Also, the stalked glands of most Byblis species are comparatively long, and would require especially copious secretions in order provide a medium by which the prey could come into contact with the digestive secretions. Moreover, in thread-like leaves which are erect or semi-erect, gravity might cause secretions to run down the leaf before enveloping the prey sufficiently to provide a connection with the leaf surface, resulting in a potentially very inefficient trapping strategy. It is also notable that there is a distinct lack of published photos of Byblis plants showing prey adhering to the outer stalks that is enveloped by sufficiently copious mucilage secretions to form a connection with the leaf surface/digestive glands. This correlates with observations made in the private collection of the author.

The possibility of motile ("active") traps in Byblis was first suggested publicly in 2009 by Cindy Chiang, an amateur horticulturalist who is very skilled at cultivating annual Byblis in her 
native Singapore. Chiang posted a photograph on the ICPS internet forum of a freshly caught fly on a $B$. guehoi specimen, and another photograph taken a few hours later which, she thought, suggested that the fly had been drawn closer to the leaf surface. Moreover, causal observations made over many years in the author's private collection suggested that it is common for plants of all species of Byblis to be seen with dry prey stuck to the leaf surface, especially on the underside of the leaves, a position in which the prey is most unlikely to have settled without some intervention from the plant. This phenomenon can also be observed in some published photographs of Byblis species in habitat (e.g. McPherson 2008, p 255 and 267; McPherson 2010, p 955; Lowrie 2013, p 204).

\section{Materials and methods}

The experiments all involved placing small fragments of animal-derived protein matter (and sometimes, for comparison, inorganic matter) on the stalked glands of specimens of all Byblis species and observing the reaction of the stalks and glands. Although the fragments were predominantly placed on the stalked glands of the leaves, some were also placed on the stalked glands of pedicels and stems. Particular care was taken to ensure that the fragments were placed in a manner which ensured that they were not in contact with the surface of the leaf, pedicel, or stem. Accordingly, none of the fragments were initially in contact with digestive glands.

Over a period of three years, experiments were repeatedly conducted on cultivated specimens of B. aquatica, B. filifolia, B. gigantea, B. guehoi, B. lamellata, B. liniflora, B. pilbarana, and B. rorida . The selected $B$. gigantea and $B$. lamellata specimens were mostly greenhouse-cultivated, whilst the annual species were mostly specimens grown indoors in a heated terrarium under fluorescent lights. The experiments involved placing small fragments of flake fish food (consisting primarily of fish, mollusc, and crustacean meal), cheese, and dried bloodworms on the stalked glands of leaves, pedicels, and stems. The varied food fragments had a maximum diameter of approximately $3 \mathrm{~mm}$. Except with $B$. aquatica (see below), the fragments were always placed on glands with long stalks so that they were the maximum possible distance from the digestive glands. Stalked glands on the underside of the leaves and (where relevant) pedicels were selected: depending on whether the leaf or pedicel in question was upright or horizontal, this was done in order to eliminate or mitigate any effects of gravity. In B. aquatica, the gland stalks are extremely short (usually well under $1 \mathrm{~mm}$ in length), so it was not possible to ensure that the fragments were any significant distance from the

digestive glands. Also, a detached wing of a small fly, and a detached and dried abdomen of another small fly, were placed in a similar fashion on glands of an adult B. liniflora specimen. The fragments were observed at regular intervals over 24-48-hour periods. Although most experiments were conducted on healthy plants, some observations were also made of plants grown in sub-optimal conditions (such as B. filifolia plants on a windowsill in November in the United Kingdom with no artificial lighting).

To compare the reaction to animal proteins and other matter, an experiment was conducted whereby ten fragments each of perlite and kitchen paper (similar in size to the fragments mentioned above) were placed on stalked glands of four greenhouse-cultivated specimens of B. gigantea and one of $B$. lamellata. The fragments were observed at regular intervals over a 72-hour period. Two of the cheese fragments were placed on old leaves which, although still producing mucilage from the stalked glands, were beginning to turn brown at the leaf tips. Similar, albeit more casual, experiments with inorganic matter were also conducted on B. filifolia, B. guehoi, B.liniflora, B. pilbarana, and $B$. rorida. 
As will be discussed below, the observations made as a result of the above experiments yielded some very surprising and interesting results. In order to scrutinize more carefully the reaction of the plants to the placement of the fragments, several time-lapse videos of stalk cell movement were produced using fragments of flake fish food, cheese, or dried bloodworms (see https://youtu.be/ xoftn_EWOz8). These fragments were, as with the previous experiments, placed on the long-stalked glands on the underside of the lamina of two-month-old specimens of B. filifolia and B. rorida, both of which were close to anthesis, a one-month-old specimen of $B$. guehoi, flowering specimens of $B$. liniflora, B pilbarana, and B. rorida, and a two-month-old seedling of $B$. gigantea. Fragments were also placed on the pedicel of a flowering B. rorida specimen, and the stem of the $B$. guehoi specimen. These specimens and species were chosen on account of being grown indoors (the annual species in an illuminated terrarium, and B. gigantea on an illuminated shelf) so that they were accessible for the purposes of creating a time-lapse video over periods of many hours. In the case of the $B$. rorida pedicel, the specimen was chosen because it was flowering at an opportune moment, and because the stalks on the pedicel were relatively long compared to those of B. filifolia (which was flowering contemporaneously in the author's collection), making the $B$. rorida pedicel a suitable subject for video observation.

\section{Results and discussion}

The observations conducted for the purposes of this article provide clear evidence of motile traps in all currently recognized Byblis species. The fragments of organic matter containing animal proteins (including the cheese, fish food, bloodworm, and the body parts of dead flies) which were placed on the stalked glands were almost invariably brought into direct contact with the leaf surface over a period of 2 to 24 hours (see, for example, the progress of the insect wing on glands of $B$. liniflora (Fig. 4). Stalked glands on pedicels and stems were observed to react in the same manner. The time-lapse videos unequivocally demonstrate that the transportation of the fragments towards the leaf surface is primarily achieved by a process whereby the stalks of glands whose gland heads are in contact with the fragments suffer a rapid loss of turgidity, causing them to collapse adaxially (i.e. in the direction of the leaf surface). The stalks of the pedicels and stems were observed to react in an identical manner. These findings support a reassessment of Darwin's categorization of the traps of Byblis as non-motile. Moreover, Byblis may be the only genus of carnivorous plant in which the stems and pedicels engage in active carnivory. The results also suggest that Byblis are able to differentiate between fragments which contain animal proteins and those which do not, for the latter are only very rarely transported to the leaf surface. This suggests that the stalk collapse is induced chemonastically (Fleischmann, pers. comm.).
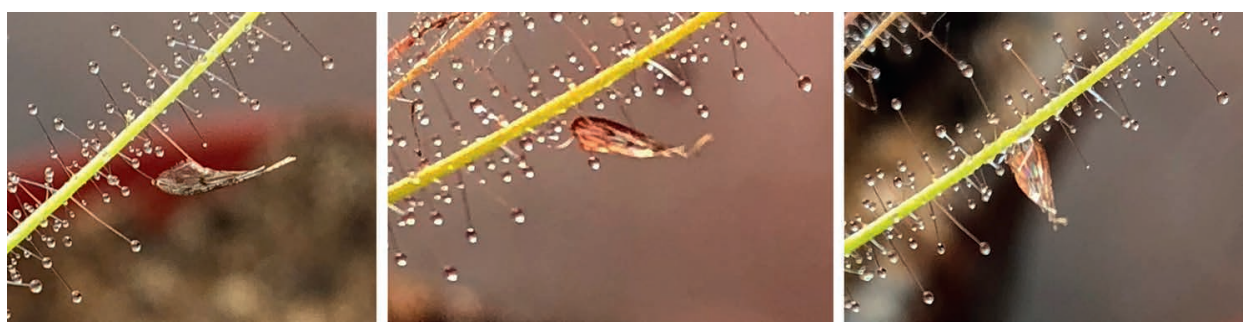

Figure 4: Cultivated Byblis liniflora with insect wing. These photos show the progress of the wing towards the leaf surface. 
Details of the process of motile trapping function in Byblis

The decision to use time-lapse video photography was motivated by the results from the set of experiments which involved intermittent observations without video technology. As well as showing fragments which had been brought into contact with the digestive secretions on the leaf surface, these intermittent observations revealed an earlier part of the process, when fragments had been drawn part of the way to the leaf surface but were not enveloped in a pool of fluid. This suggested that capillary action was probably not primarily responsible for drawing the fragments towards the digestive glands and raised a strong suspicion of a motile trapping process. The time-lapse video photography provides conclusive evidence of stalk movement in response to the capture of organic matter containing animal proteins. The videos (https://youtu.be/xoftn_EWOz8) show that glandular reaction usually begins within a period of approximately two hours, although it can commence within mere minutes or only after several hours have elapsed. Movement of the stalks can usually be observed to be directly preceded by secretions from the digestive glands around the base of the affected stalked glands. Next, the stalks appear to collapse towards the leaf axis, drawing the fragment towards the leaf surface. Once in close proximity to the digestive glands on the leaf surface, the fragment makes contact with the pooled digestive secretions, and the resulting capillary action appears to accelerate the final part of the process. Depending on the size and weight of the food fragment and the species of Byblis, one to four hours were usually required until contact with the leaf surface. After contact occurs, the fragment is enveloped for many hours or longer by the fluid secreted by the digestive glands. Again, the process appeared to be identical for stalks on the pedicels and stems.

Given that there is no perceptible increase in the secretions from the stalked glands during the motile phase (which, if present, would suggest that the collapse is caused by movement of fluid from the stalk cells to the stalked glands (Fleischmann, pers. comm.)), it would seem to be the case that, upon stimulation by prey, fluid contained in the stalk cells passes into reservoir cells positioned at the base of the respective stalk cell, thus causing the collapse of the latter. It is perhaps pertinent to mention that Lloyd (1942, p 97) notes that each stalked cell "stands on a group of basal cells which may be as many as eight in number". It may be that these basal cells serve as reservoirs to facilitate the collapse of the stalk cells. Should this explanation be correct, the process of motile trapping outlined here would appear to rely on communication between the stalked glands and the stalk cells, reservoir cells and sessile glands (Fleischmann, pers. comm.).

The movement of the stalks is, when observed through a time-lapse video, jerky rather than uniform. Food fragments that are in contact with more than one stalked gland are often twisted and partially rotated as they are moved. When a fragment is adhered to a single long-stalked gland, the stalk can be seen to flatten, and the fragment performs multiple 360-degree rotations as it travels towards the leaf surface. This suggests that the stalks rotate in a spiraliform manner as they collapse. Interestingly, Lloyd (1942, p 97) describes how, when a stalked gland of Byblis "dries (in air or alcohol), the stalk cells twist." Moreover, Darwin (1875, p 344) notes that the walls of these elongated cells are "marked with the most delicate spiral lines", and that dried stalk cells are invariably found to be "spirally rolled up". Fenner (1904), who studied the morphology of B. gigantea in detail, noted the spiral structure, and thought it an adaption "to allow bending of the [stalk] without collapse" (as translated by Lloyd (1942, p 97). But the observations conducted in connection with this work clearly show that the stalk cells do collapse, apparently permanently so. The presence of the spiral lines provides a convincing explanation for the spiraliform collapse of the stalks that is evident in the time-lapse videos.

Stalk movement which conveys the fragments towards the leaf surface, or that of the pedicels or stalks, is only observed in stalks whose glands come into contact with the animal proteins. There is 
no evidence of any reaction by stalked glands in the vicinity of those to which fragments are adhered which might serve to assist the latter in transporting the fragments. Observations made several hours after collapse of the selected stalked glands do, however, suggest that copious secretions from the digestive glands can precipitate further collapses of nearby stalked glands. But these collapses almost invariably occur once the fragment is in contact with the digestive secretions and play no role in transportation of the fragments. Motility, therefore, seems to be restricted to those glands that are in direct contact with the food fragments. It was also observed that the reaction of the stalks is much reduced or even absent in very old leaves and pedicels, and in plants grown in sub-optimal conditions.

Given the lack of any evidence that the stalked glands produce additional secretions in response to prey capture, it can be observed that the digestive glands appear to be solely responsible for all additional secretions which eventually envelop the fragments. Gradual reduction in the size of the organic fragments can be observed in the hours or even (usually in the case of the perennial species) days after contact with the secretions from the digestive glands, which is suggestive that digestive enzymes are present in these secretions. Additionally, it appears that, once a stalk cell has collapsed, it becomes spent, and does not regain its turgidity. This indicates that the process is connected to desiccation of the stalks and is quite unlike any motility exhibited by the traps of any other carnivorous plants (Fleischmann, pers. comm.). Certainly, observations made several days after apparent completion of the digestive process have provided no evidence of any restoration of the turgidity of the stalked glands. But, at least in the annual B. filifolia, B. liniflora, and B. rorida, the useful life of each leaf is little more than a month (Bourke, pers. comm., also the author's own observations on cultivated specimens). In these species, there would appear to be little cost benefit in cells possessing the ability to reset themselves for further carnivory. So, this trait might perhaps be particularly suited to the annual species, all of which exhibit rapid seasonal growth.

The results of the experiments conducted for the purpose of this article provide evidence as to why plants with access to insect prey can be seen frequently with dried prey attached to the leaf, pedicel, and stem surfaces. The ability of the stalked glands to collapse is very likely to improve the efficiency of the carnivorous syndrome in Byblis by bringing captured prey within range of the secretions of the sessile digestive glands. It would also seem reasonable to suppose that the motile abilities of the stalks may in many cases serve to bring captured prey into contact with other nearby stalked glands, thus enhancing the efficiency of the manner in which prey is ensnared (Rivadavia, pers. comm.). Nevertheless, the type of motile carnivorous trap outlined in this article may be regarded as rather simple compared to the far more sophisticated and strikingly different version practiced by Drosera (see generally Lloyd 1942). In particular, the stalks of Byblis, like those of other carnivorous Lamiales, but unlike those of carnivorous Caryophyllales such as Drosera, have no vascular bundles (Fleischmann et al. 2018; Lloyd 1942; Juniper et al. 1989), and cannot deliberately bend towards a chemical or other stimulus. The stalks of Byblis do not work in a coordinated manner, and observations suggest that an inconveniently placed leg or wing may prevent captured prey from being dragged into an optimal position for digestion and nutrient assimilation. It might, however, be noted that Setocoris bugs are likely to assist Byblis plants in sequestering nutrients from parts of the prey that remain outside of the range of the digestive fluids (Fleischmann, pers. comm.).

Evidence that motility in Byblis is triggered by detection of animal proteins rather than mechanical stimulation

Despite the generally simple nature of the motile trapping exhibited by Byblis, observations indicate that the plants may be able to detect, and react accordingly to, the capture of animal proteins. 

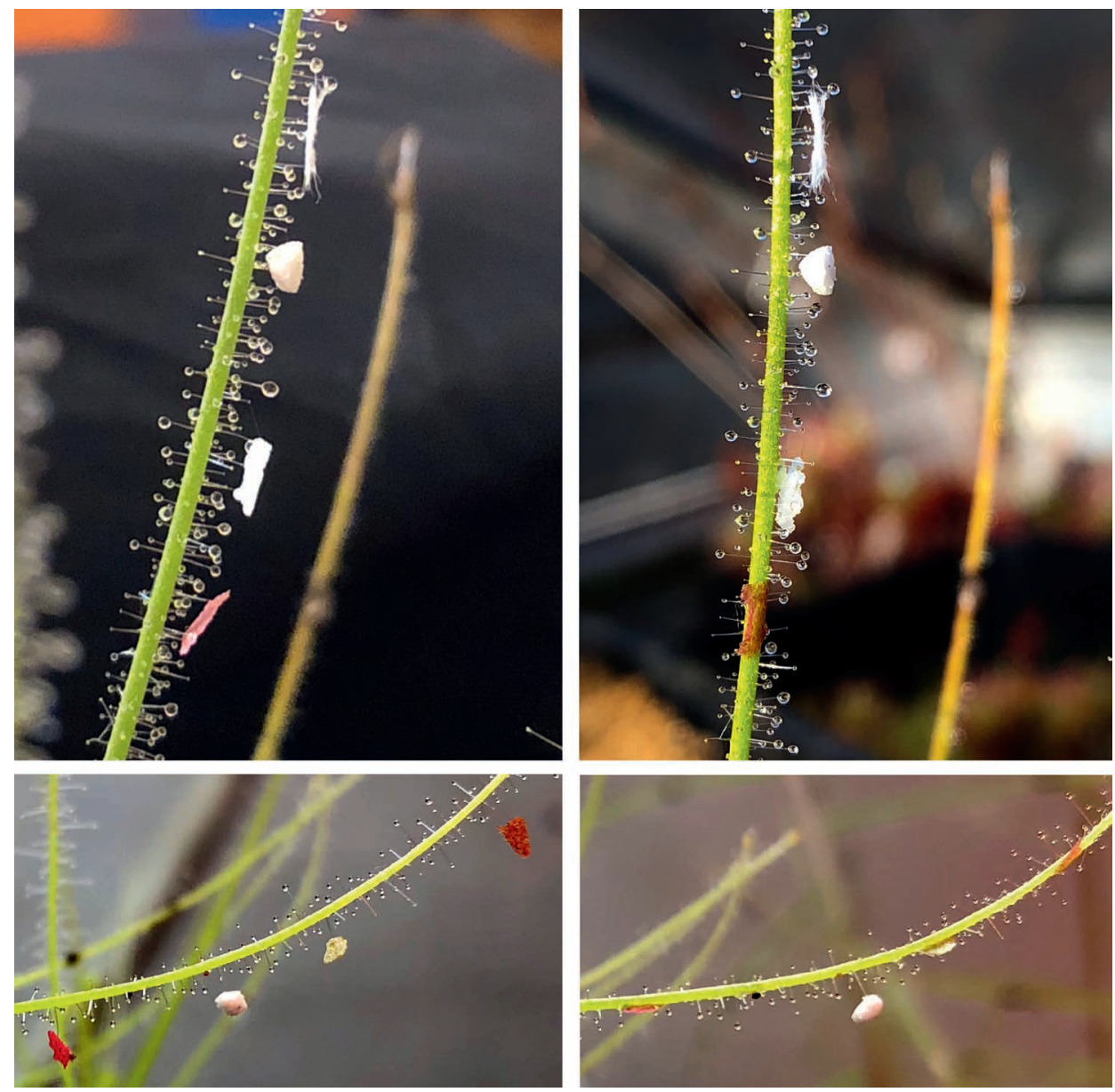

Figure 5: Top: Cultivated Byblis gigantea with (top to bottom) paper, perlite, cheese, and fish food adhered to stalked glands. Bottom: Cultivated Byblis filifolia with (left to right) fish food, perlite, fish food, and fish food adhered to stalked glands. Note how the perlite and paper show no signs of movement towards the leaf surface.

Unlike the fragments of animal proteins, similarly sized fragments of perlite and kitchen paper usually elicit no response from the stalks (see Fig. 5 top: B. gigantea with (from top to bottom of leaf) kitchen paper, perlite, cheese, and fish food; Fig. 5 bottom: B. filifolia with (from left to right of leaf) fish food, perlite, fish food, and fish food). This suggests that it is chemical rather than mechanical stimuli which provoke movement in Byblis. This effect is shown clearly by the results of the experiment conducted on B. gigantea and B. lamellata. None of the ten fragments of perlite or the ten of kitchen paper showed any evidence of movement, whilst the ten fragments of fish food all migrated to the leaf surface of the same plants. Of the ten fragments of cheese, eight had migrated to the leaf surface, whilst it was noted that both static fragments of cheese were positioned on the old leaves. Similar, albeit more casual, observations have been made on several occasions using the perennial species and also B. filifolia, B. guehoi, B. liniflora, B. pilbarana, and B. rorida. 
It should, however, be noted that occasional observations, particularly on B. filifolia, B. pilbarana, and $B$. rorida, were made of perlite fragments having been transported to the leaf surface. These observations have virtually always been observed in respect of fragments of perlite placed in close proximity to fragments of organic matter containing animal proteins, and the reaction to the perlite was always far slower than the reaction to the organic fragments. It is here pertinent to recall the observations of the delayed collapse of stalk cells in the vicinity of copious secretions from the digestive glands. It is tentatively suggested that the occasional transport of the perlite to the leaf surface is a result of this latter phenomenon and does not represent a deliberate reaction to the capture of the inorganic material.

Interestingly, it has been observed that in nature, the collapse of stalked cells is not caused by the Setocoris bugs which often inhabit the plants as commensals (Bourke, pers. comm.). Similar observations have been made in cultivated specimens of B. filifolia, B. gigantea, B. guehoi, and B. lamellata which play host to Setocoris in the private collection of the author of this paper. This may be consistent with the notion that the stalk cell collapses occur primarily in response to detection by the stalked glands of chemical stimuli in captured animal proteins rather than to what is commonly referred to as a sense of touch.

Evidence of intra-specific differences in the process of active carnivory in Byblis

The observations conducted for this paper suggest that $B$. aquatica probably benefits very little, if at all, from its motile traps. This species, at least when beyond the seedling stage, produces extremely short stalks whose glands are furnished with relatively large droplets of mucilage. The secretions from the digestive glands (which are particularly copious), together with the large mucilage droplets from the stalked glands, are easily able, without any apparent need for stalk movement, to envelop materials which become adhered to the stalked glands (see Fig. 6). Indeed, in this respect, B. aquatica appears to employ a method of carnivory that superficially bears more similarity to that of Drosophyllum than to that of its fellow Byblis species (albeit that the precise mechanisms are not at all similar, because of the vascularization of the trapping organs in Drosophyllum). Perhaps notably, similar to Drosophyllum but unlike many if not all annual Byblis species, B. aquatica does not present stalked glands along the midrib (Bourke, pers. comm.).

The other species of Byblis, all of which have stalks which are much longer on average than those of $B$. aquatica, appear to be essentially uniform in their mode of carnivory. Some small differences were, however, observed. Insofar as the spiraliform stalk collapse is concerned, only partial rotations of the fragments can be observed in species with relatively short stalks, most notably B. pilbarana. This contrasts with longer-stalked species, such as $B$. liniflora, in which multiple 360-degree rotations always occur. The annual species of Byblis exhibit the most consistently rapid reactions, the prey usually being conveyed into contact with the leaf surface over a period of between two and six hours. The fastest reactions were observed in B. rorida, where stalk contraction can commence within a matter of minutes, and the entire process of transporting the fragment to the leaf surface can be completed within less than one hour of the placement of the fragment on the stalked gland. Intriguingly, $B$. rorida, has been shown by Laura Skates to be especially reliant, when compared to B. filifolia, B. gigantea, and B. liniflora, upon carnivory for obtaining nitrogen (Skates 2016).

With the perennial species, observation of the process is complicated somewhat by the overwhelming propensity of the glands to react only outside of daylight hours to the capture of prey. The process of drawing prey towards the leaf surface typically begins after sunset, and digestive fluid is almost exclusively secreted and re-absorbed overnight, so that, a few hours into the morning, unless the weather is particularly overcast, the glands are dry, and the prey is stuck fast to the surface of the 

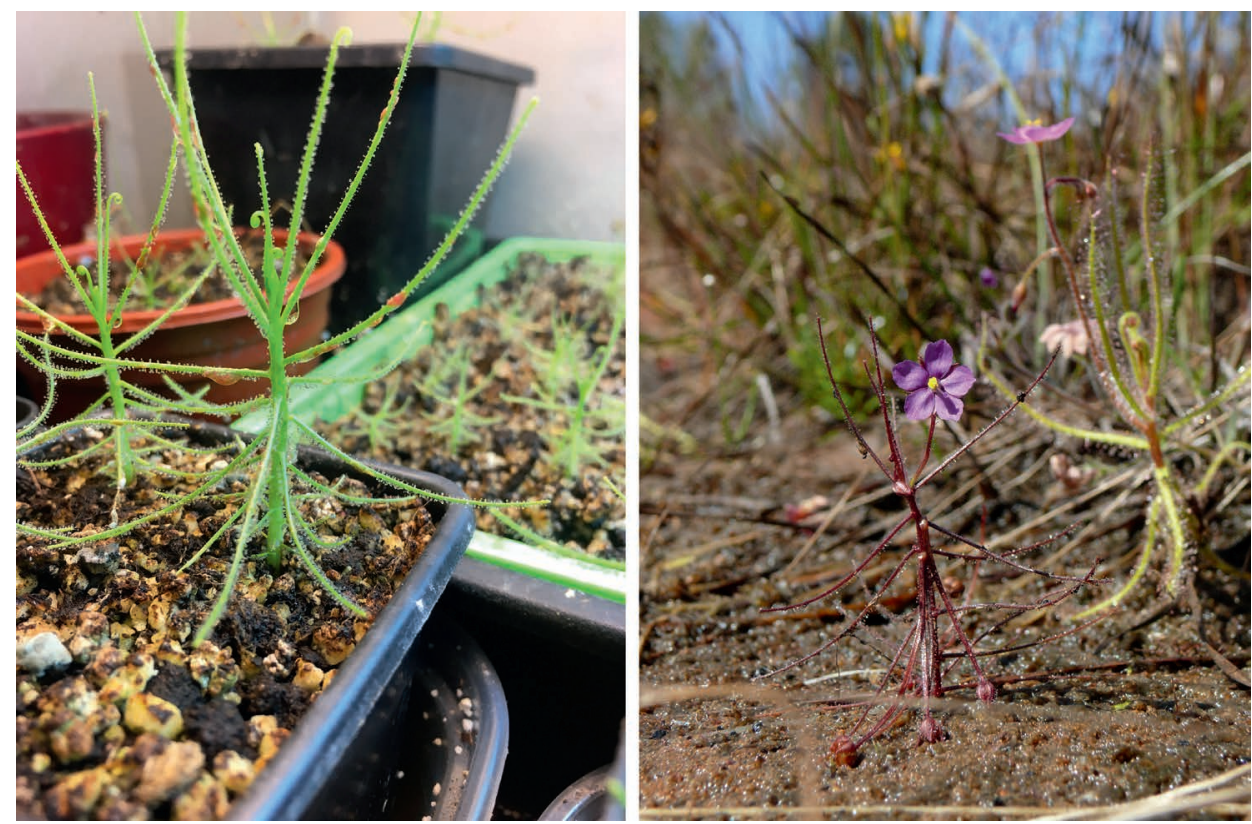

Figure 6: Cultivated (left) and wild (right, image courtesy of Andreas Fleishmann) Byblis aquatica showing very short stalks. Note the relative size of the pooled digestive fluid in the left photo.

leaf, stem, or pedicel. The process of secretion and absorption, if necessary, can be repeated over successive nights until the digestive process is complete.

\section{Further research}

It should be noted that since the observations explored in this paper were first made publicly available (via Facebook), horticulturalist and botanist Siggi Hartmeyer, has repeated the results in B. liniflora and B. filifolia, and in a hybrid between the two species (Hartmeyer \& Hartmeyer 2019, p 74 this issue), capturing some extremely detailed video footage (https://youtu.be/2hoUCQjf2LE) of the cellular collapse. Hartmeyer also observed the phenomenon in a young B. aquatica plant, but he noted that in this species, the motile ability of the stalk cells was weak, and was unable to convey even a small fragment of fish food to the leaf surface without the assistance of gravity (pers. comm.). This supports the suggestions in this paper that the mechanism of carnivory in B. aquatica differs from that employed by other species in the genus. Another skilled cultivator of Byblis in the United States of America, Anthony Bell, has also observed the apparent transportation of flake fish food from the outer stalked glands to the leaf surface in B. gigantea (pers. comm.).

There is much scope for future investigations into the carnivorous syndrome in this under-studied genus. In particular, further research is required into the means by which the plants detect matter that is suitable for digestion and the factors which cause the stalk cells to collapse. It would also be very useful to investigate the manner in which the cells in the stalked glands, stalk cells, and digestive glands communicate with one another and co-ordinate their response to prey capture. A very fruitful avenue for further research would be to insert dye into the stalk cells to determine the 
direction of the fluid diffusion which causes the collapses (Fleischmann, pers. comm.). The precise type of carnivory employed by B. aquatica, and its relationship in this respect with other species within the genus, should also be the subject of future studies, as should investigation into whether there is any correlation between the speed of the reaction of the stalked glands in particular species and the extent of their reliance upon carnivory for nutrient sequestration. It would also be potentially fruitful to assess the motility of the traps throughout the life cycles of various species. Finally, of course, it would be of great benefit if other horticulturalists, and in particular those with access to wild populations of Byblis, could seek to repeat the results outlined in this paper.

\section{Conclusion}

The observations in this article provide convincing evidence that Byblis ought no longer to be regarded as a genus of carnivorous plants with non-motile traps. Upon coming into contact with suitable prey, the stalk cells of the leaves, stems, and pedicels collapse inwards in a spiral motion, thus drawing prey towards the digestive glands and within range of the digestive secretions. This, it is submitted, provides strong evidence that all currently described members of the genus Byblis possess motile adhesive traps and should henceforth be treated as "active carnivores".

Acknowledgments: The author would like to thank Anthony Bell, Colin Bourne, Siggi Hartmeyer, Allen Lowrie, and Paul Temple for their input into various discussions about the findings presented in this article. The author owes a particular debt of gratitude to Greg Bourke, Andreas Fleischmann, and Fernando Rivadavia for their advice, and for their comments on earlier drafts of this paper. The author is also especially grateful to Greg Bourke and Andreas Fleischmann for providing photographs and giving the authorization to publish them in this article (Greg Bourke provided Fig. 3 (left); Andreas Fleischmann provided Figs. 2, 3 (right), and 6 (right)). Finally, much credit must go to Cindy Chiang as the person who suggested to the author the possibility that Byblis might employ movement in its carnivorous process. Of course, notwithstanding the invaluable contributions of the above-named persons, any errors in this article should be attributed to the author alone.

\section{References}

Allan, G. 2019. Video: "Evidence of motile traps in Byblis". https://youtu.be/xoftn_EWOz8

Anderson, B., and Midgley, J.J. 2003. Digestive mutualism, an alternate pathway in plant carnivory. Oikos 102: 221-224.

Bauer, U., Jetter, R., and Poppinga, S. 2018. Non-motile traps. In: Ellison, A.M., \& Adamec, L. (eds.): Carnivorous Plants: Physiology, Ecology, and Evolution. Oxford University Press: 194206.

Bourke, G., and Nunn, R. 2012. Australian Carnivorous Plants. Redfern Natural History Productions. Poole, Dorset, England: 1-197.

Bruce, A.N. 1905. On the activity of the glands of Byblis gigantea. Notes of the Royal Botanic Garden of Edinburgh 16: 89-98.

Cross, A.T., Paniw, M., Scatigna, A.V., Kalfas, N., Anderson, B., Givnish, T.J., and Fleischmann, A. 2018. Systematics and evolution of small genera of carnivorous plants. In: Ellison, A.M., \& Adamec, L. (eds.): Carnivorous Plants: Physiology, Ecology, and Evolution. Oxford University Press: $120-134$.

D’Amato, P. 1998. The Savage Garden: Cultivating Carnivorous Plants. Ten Speed Press. Berkeley, California: 1-314. 
Darwin, C. 1875. Insectivorous Plants. D. Appleton and Company. New York: 1-462.

Fenner, C.A. 1904. Beiträge zur Kenntnis der Anatomie, Entwickelungsgeschichte und Biologie des Laubblätter und Drüsen eniiger Insektivoren. Flora 93: 335-434.

Fleischman, A., Schauler, J., Smith, S.A., and Givinish, T.G. 2018. Evolution of carnivory in angiosperms. In: Ellison, A.M., \& Adamec, L. (eds.): Carnivorous Plants: Physiology, Ecology, and Evolution. Oxford University Press:22-41.

Hartmeyer, I., and Hartmeyer, S. 2005. Byblis filifolia als echte Karnivore rehabilitiert. Das Taublatt 53: 4-5.

Hartmeyer, I., and Hartmeyer, S.R.H. 2018. Video: "Byblis and Lindernia: Motion and enzyme tests". https://youtu.be/2hoUCQjf2LE

Hartmeyer, I., and Hartmeyer, S.R.H. 2019. Observations and tests on cultivated tropical Byblis. Carnivorous Plant Newsletter 48: 74-78.

Hartmeyer, S. 1997. Carnivory of Byblis revisited: a simple method for enzyme testing on carnivorous plants. Carnivorous Plant Newsletter 26: 39-45.

Hartmeyer, S. 1998. Carnivory in Byblis revisited II: the phenomenon of symbiosis on insect trapping plants. Carnivorous Plant Newsletter 27: 110-113.

Lloyd, F. 1942. The Carnivorous Plants. Waltham. Massachusetts: 1-352.

Lowrie, A. 1998. Carnivorous Plants of Australia, Volume 3. University of Western Australia Press. Nedlands, Western Australia, Australia: 1-288.

Lowrie, A. 2013. Carnivorous Plants of Australia Magnum Opus, Volume 1. Redfern Natural History Productions. Poole, Dorset, England: 1-458.

McPherson, S. 2008. Glistening Carnivores, The Sticky-Leaved Insect-Eating Plants. Redfern Natural History Productions. Poole, Dorset, England: 1-392.

McPherson, S. 2010. Carnivorous Plants and their Habitats, Volume 2. Redfern Natural History Productions. Poole, Dorset, England: 725-1442.

Płachno, B.J., Adamec, L., Lichtscheidl, I.K., Peroutka, M., Adlassnig, W., and Vrba, J. 2006. Fluorescence labelling of phosphatase activity in digestive glands of carnivorous plants. Plant Biology 8: 813-820.

Płachno, B.J., and Muravnik, L.E. 2018. Functional anatomy of carnivorous traps. In: Ellison, A.M., \& Adamec, L. (eds.): Carnivorous Plants: Physiology, Ecology, and Evolution. Oxford University Press: 167-179.

Rice, B. 2006. Growing Carnivorous Plants. Timber Press. Portland, Oregon: 1-224.

Skates, L., Dixon, K., Gebauer, G., Stevens, and Cross, A. 2016. Carnivorous Plants. Poster at the $11^{\text {th }}$ ICPS Conference Kew, UK, 5-7 August 2016.

Slack, A. 1979. Carnivorous Plants. Marston House. Yeovil, Somerset, England: 1-240.

Slack, A. 1986. Insect-Eating Plants and How to Grow Them. Alphabooks. Sherborne, Dorset, England: 1-172. 